\title{
Data of food challenges of an outpatients' pediatric allergy clinic in the Mediterranean island of Crete, Greece
}

\author{
Evangelia Stefanaki*, Georgia Chatzitzanou, Maria Anatoliotaki, Sofia Stefanaki, Vassiliki Aggelakou \\ From Food Allergy and Anaphylaxis Meeting 2014 \\ Dublin, Ireland. 9-11 October 2014
}

\section{Introduction}

Food allergy is defined as an adverse immunologic response to a dietary protein. Diagnosis involves a careful history and diagnostic tests, such as skin prick testing, serum-specific immunoglobulin E (IgE) testing and, if indicated, oral food challenge which is the gold standard of diagnosis.

\section{Methods}

We studied retrospectively the food challenges organized by our Outpatients Paediatric Allergy Clinic between 2011-2013 and we recorded the culprit food allergens, age at challenge, challenge outcome, diagnostic work-up before challenge and coexistence of eczema.

\section{Results}

We recorded 38 children that underwent 75 challenges. The median age at challenge was 15 months and $68,4 \%$ $(26 / 38)$ of the children were boys. $50 \%(14 / 28)$ of the children had a family history of atopy. $66 \%(25 / 38)$ of the children were diagnosed with atopic dermatitis at the time of reaction or/and at diagnosis. 73\% (19/26) of the children with atopic dermatitis had only mild persistent signs of the disease. $68,4 \%(26 / 38)$ of all the children had a history of clinical reaction (in $77 \%, 20 / 26$ of the patients the reaction was immediate) and the median age at first reaction was 6 months while 31,6\% (12/38) were only sensitized to the allergen of the challenge. $52,6 \%(20 / 38)$ were sensitised or reacted to only one allergen most commonly to egg (6/20) and milk (6/20). We recorded 27 (36\%) challenges to milk- 7 to partially hydrolysed milk (after negative prick-to-prick PTP test to pHA milk), 10 to evaporated milk, 4 to common formula, 3 to cake with milk, 2 to fresh milk and 1 to yoghurt. At the time of milk challenges the average diameter for prick to prick test was $5,1 \mathrm{~mm}$ for $\mathrm{HA}$, $3,6 \mathrm{~mm}$ for evaporated milk and $7,3 \mathrm{~mm}$ for cake only with milk. We also recorded $22(29,7 \%)$ challenges to egg (7/22 to cake with egg). The average PTP was 1,8 $\mathrm{mm}$ to boiled egg white and $0,84 \mathrm{~mm}$ for egg yolk. We finally recorded 6 challenges to fish, 6 to wheat, 2 to lentils and 1 to potato. Only $1 / 75$ challenges was positive and the reaction was only mild.

\section{Conclusions}

Our food challenges under safe conditions placed no patient at risk for serious reactions. Atopic dermatitis although mild is still a major risk factor for food allergy. Different types of milk fit differently to each milk allergic patient.

Published: 30 March 2015

Venizelion General Hospital, Heraklion, Crete, Greece 